\title{
Integrating Passive UHF RFID Tags with WSN Nodes: Challenges and Opportunities
}

\author{
Danilo De Donno, Maria Laura Stefanizzi, Luca Catarinucci, Luca Mainetti, Luigi Patrono, and Luciano Tarricone
}

\begin{abstract}
Radio Frequency Identification (RFID) and Wireless Sensor Networks (WSNs) have received an everincreasing attention in recent years, mainly because they represent two of the most important technologies enabling the Internet of Things vision. Although designed originally with different objectives, WSN and RFID represent two complementary technologies whose integration might increase their functionalities and extend their range of applications. However, important technological issues must still be solved in order to fully exploit the potentialities offered by such integration. In this work, an innovative RFID-WSN integration approach is presented and validated. It relies on the interconnection of a new-generation, long-range, EPCglobal Class-1 Generation-2 Ultra-High-Frequency (UHF) RFID tag with a commercial WSN node via the $I^{2} C$ interface. Experimental results have demonstrated the effectiveness of the proposed approach compared to existing solution in the literature. Interesting application scenarios enabled by the proposed RFIDWSN integration approach are briefly summarized at the end of the paper.
\end{abstract}

Index Terms-RFID, Wireless Sensor Network, UHF, integration approach, wake-up radio.

\section{INTRODUCTION}

$\mathrm{T}$ HE Next Generation Internet (NGI) aims at the integration of heterogeneous communication technologies, both traditional and emerging, in order to assert the so-called Internet of Things (IoT) vision [1]. According to this concept, everyday objects, such as domestic appliances, actuators, and embedded systems of any kind, will become proactive actors of the Internet, with the capability to generate and consume information. In such a new vision, devices will be able to interact with both users and objects, making available a great amount of data, previously inaccessible, about the physical world. By exploiting the new possibilities offered by IoT enabling solutions, innovative smart applications are being developed in many interesting scenarios.

Several new technologies and standards are leading this evolutionary trend. Among those, Radio Frequency Identification (RFID) and Wireless Sensor Networks (WSNs) represent two of the most promising solutions. RFID is a very low-power technology mainly characterized by passive devices, called tags, which are able to send stored data when

Manuscript received March 25, 2014, revised May 20, 2014.

Authors are with the Innovation Engineering Department, University of Salento, Via per Monteroni, 73100, Lecce, Italy.

L. Patrono is the corresponding author (e-mail: luigi.patrono@ unisolento.it). powered by the electromagnetic field generated by a reader. Such devices are usually classified according to the frequency band used (e.g. LF, HF, UHF, etc.) and the type of coupling (i.e. magnetic or electromagnetic) between tag and reader antennas. The main strengths of this technology are cost effectiveness, small size and long lifetime of the tags. This last feature is particularly noteworthy. Indeed, since passive tags do not need a source of energy to operate, their lifetime can be measured in decades, thus making the RFID technology well suited for many industrial applications, such as warehouse management, objects localization and tracking [2]. However, since passive tags can operate solely under the reader coverage area, the use of RFID technology is limited to the identification of objects in small areas. The integration of RFID and WSN technologies can overcome this limit, enabling the development of complex next-generation applications. Basically, WSNs consist of a large number of low-cost, low-power embedded devices, called sensor nodes, which are able to self-configure and self-organize. These characteristics make them suitable to be deployed even in harsh environments in order to detect important parameters (e.g. temperature, light, humidity, etc.) without human intervention. The collected data are delivered, in a multi-hop fashion, to a central point (sink) for proper utilization. This simple, yet fundamental functionality, has enabled the widespread adoption of WSN technology in many heterogeneous scenarios, such as building automation, surveillance, military operations, healthcare, logistics, etc. However, one of the main issues to be addressed in the development of complex applications is the power consumption. Indeed, sensor nodes are usually battery powered and deployed in large areas where changing or replacing batteries could be impractical or completely unfeasible. Therefore, energy consumption is a primary issue to be considered, and the use of effective solutions to increase the network lifetime is fundamental in real applications [3][7].

Although RFID and WSN were originally designed with different objectives, the benefits provided by both technologies suggest the development of an integrated solution, able to combine identification (RFID) and advanced communication (WSN) capabilities [8]. Such an integration could maximize the effectiveness of both technologies, giving new perspectives to a broad range of novel applications. By merging RFIDs with WSNs important information such as object identity and location, but also environmental conditions, could be retrieved. In such a vision, for instance, a products along with their environmental conditions. Moreover, RFID systems integrated with WSNs could benefit from the 
multi-hop wireless communication, thereby overcoming the main drawback of the RFID technology, i.e. the single-hop communication with the reader.

In the last years, several attempts to integrate WSN and RFID technologies have been proposed, mainly with the aim of providing innovative services. A first example is presented in [9] where a wireless localization system for monitoring children position in theme park is implemented by equipping WSN nodes with RFID reader capabilities. The localization problem is also addressed in [10], where a grid of RFID tags is used to enhance the localization accuracy of standard RSSIbased WSN algorithms. In [11], authors propose the integration of WSN nodes and RFID readers for the development of a smart warehouse management system. Although the integration of readers and sensor nodes gives new perspectives in likely applications, some important drawbacks exist, mainly related to the high cost of RFID readers and their high power consumption. Interesting opportunities are also offered by the integration of RFID tags with sensor nodes. In such a scenario, the RFID tag can be used to provide location-aware services and minimize the overall power consumption by exploiting the capability to wake up WSN nodes when triggered by RFID readers. Currently, only few studies have considered this approach. An RFID wake-up mechanism, namely RFIDImpulse, is proposed in [12]. It uses an IEEE 802.15.4 transceiver as a fake RFID reader able to trigger an RFID tag and, indirectly, to wake up the associated WSN node. However, authors use a coil instead of a real RFID tag for their proof of concept. To the best of our knowledge, the first real implementation of a passive wake-up radio device providing both broadcast-based and IDbased wake-ups is the WISP-Mote [13]. It integrates a WISP (Wireless Identification and Sensing Platform) [14], a Computational RFID (CRFID) tag developed by Intel Research, and a TMote Sky [15] WSN node. The main drawback of the proposed implementation is the extremely small wake-up range achieved (approximately 4 meters). As the reported works suggest, important technological issues must still be addressed in order to fully exploit the potentialities offered by the RFID-WSN integration.

In this work, an innovative RFID-WSN integration approach, based on the use of the $\mathrm{I}^{2} \mathrm{C}$ interface, is presented and validated. Specifically, a new-generation, long-range, EPCglobal Class-1 Generation-2 (Gen2 for short hereafter) Ultra-High-Frequency (UHF) RFID tag has been connected to a commercial WSN node, enabling the communication between the two devices. In order to validate the proposed approach, the energy consumption of the developed tag, in the communication phase with the WSN node through the $\mathrm{I}^{2} \mathrm{C}$ interface, has been thoroughly analyzed. Moreover, the prototyped RFID tag has been successfully used as a fully passive wake-up radio for WSN nodes and the achieved wakeup ranges in an indoor scenario have been measured. Experimental results demonstrate that our approach substantially outperforms the solutions already presented in literature in terms of maximum communication distance, thus addressing the problem of reduced coverage RFID-WSN due to the reduced operating range of RFID tags compared to that of conventional WSN nodes. Such a problem is one of main limits to the widespread adoption of RFID-WSN integrated solutions in real world applications. Finally, potential application scenarios enabled by the proposed RFIDWSN integration approach are envisioned at the end of the paper.

The rest of this paper is organized as follows. The proposed RFID-WSN integration is discussed in Section II. Next, several experiments aimed at assessing the performance of the integrated solution are presented in Section III, while potential applications are discussed in Section IV. Finally, conclusions are drawn in Section V.

\section{Proposed RFID-WSN integration}

The proposed RFID-WSN integration approach is simple yet effective and it is outlined in Fig. 1: a conventional UHF RFID tag featuring the $\mathrm{I}^{2} \mathrm{C}$ interface is physically connected to the microcontroller unit (MCU) of a WSN node. In this way, information exchanged between the tag and the RFID reader via the EPCglobal Class-1 Generation-2 (Gen2) air interface are directly available also on the $\mathrm{I}^{2} \mathrm{C}$ interface and, therefore, accessible by the MCU. Potentially, the proposed RFID-WSN integration enables EPCglobal standardized data to be spread, in a multi-hop fashion, throughout an IEEE 802.15.4 network. Details about the hardware and software components making up the outlined RFID-WSN integration approach are provided in the following subsections.

\section{A. Hardware components}

The RFID Section, highlighted in Fig. 1, schematically represents the Printed Circuit Board (PCB) prototype shown in Fig. 2. It has been fabricated in our labs by using a photolithography process on FR4 substrate and handy soldering off-the-shelf discrete components. The developed board is equipped with a battery holder (not exploited in the proposed RFID-WSN integration since the required power is fed directly from the $3-\mathrm{V}$ battery of the WSN node) enabling a stand-alone mode of operation as a battery-assisted passive (BAP) RFID tag [16].

The designed dipole-like UHF RFID antenna is patterned directly on the PCB. The input impedance of the antenna has been tuned to achieve a complex conjugate impedance matching with an Impinj Monza X-2K RFID chip [17] $\left(Z_{\text {chip }}=R_{\text {chip }}+j X_{\text {chip }}=20.83-j 181.39 \Omega\right)$ at $866.5 \mathrm{MHz}$, i.e. the center frequency of the European UHF RFID band. Monza X$2 \mathrm{~K}$ is a new-generation UHF RFID Gen2 integrated circuit (IC) with 2176 bits of non-volatile memory (NVM) and an $\mathrm{I}^{2} \mathrm{C}$ interface. As an $\mathrm{I}^{2} \mathrm{C}$ device, Monza $\mathrm{X}-2 \mathrm{~K}$ operates as a standard EEPROM whose contents can also be accessed via the Gen2 air interface. In the fabricated prototype, the small female header, which exposes the $\mathrm{I}^{2} \mathrm{C}$ bus, is used to interface the RFID chip with the WSN Section thus physically realizing the RFID-WSN integration sketched in Fig. 1.

The antenna design takes cue from the commercial ALN9660 RFID tag inlay [18] which uses meander lines to achieve a very compact form factor (i.e., $7.5 \times 1.7 \mathrm{~cm}^{2}$ ). Detailed simulations taking into account the effect of the DC metal traces have been carried out in order to maximize the performance and optimize the design of the final prototype. 


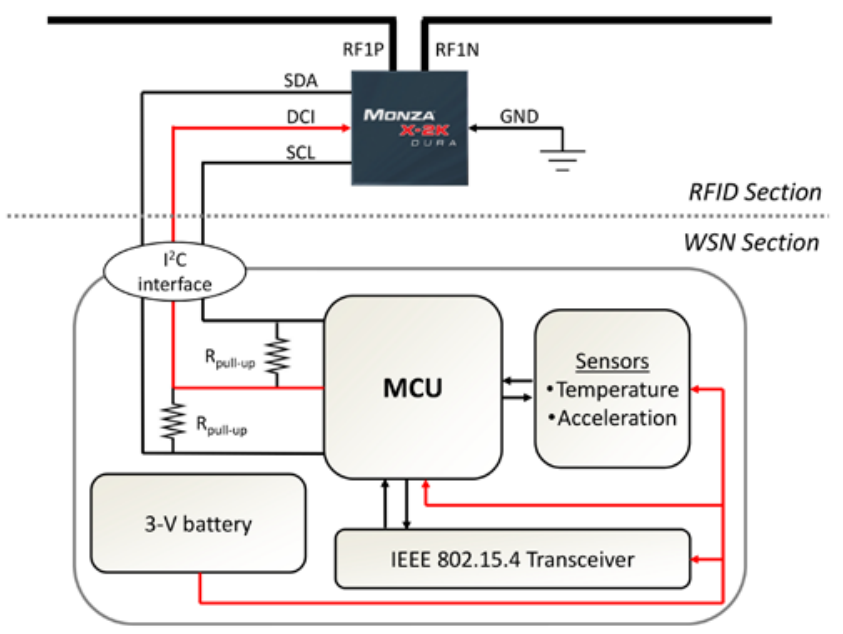

Fig. 1. Proposed integration approach between RFID and WSN. Red connections show how the on-board power is routed to the functional blocks.

Three main elements define the antenna structure: (i) the central loop, which primarily impacts on the tuning of the real part of the input impedance and prevents potential highvoltage discharge, (ii) the meander lines to reduce the antenna size, and, finally, (iii) the capacitive loads at the antenna tips, which facilitate the impedance matching. Simulations were performed by the CST MW Studio setting the input impedance of the antenna port equal to $\mathrm{R}_{\text {chip }}$ and adding a capacitive lumped element with reactance $\mathrm{X}_{\text {chip }}$. In this way, the problem is led back to the design of classic antennas with real impedance. Finally, the antenna design has been optimized by setting the minimization of the reflection coefficient at the desired frequency as the fitness function and by adopting a gradient-based interpolated quasi-newton optimizer.

The E-plane polar diagram in Fig. 3(a) depicts the typical dipole radiation pattern $(1.8 \mathrm{dBi}$ is the realized gain) achieved for the antenna while the good impedance matching around the frequency of interest $(-18-\mathrm{dB}$ reflection coefficient at 866.5 MHz) is highlighted in Fig. 3(b). A detailed comparison between simulation and measurement results for the proposed RFID tag antenna is reported in [19]. Note that, depending on the application and the harshness of the environment, a directive patch antenna instead of an omnidirectional dipole could be used to achieve higher performance (e.g. a longer read range) and platform tolerance [20], [21].

The RFID board is interfaced via the $\mathrm{I}^{2} \mathrm{C}$ bus to the MB851 board developed by ST Microelectronics [22] (see Fig. 4). This board (representing the WSN Section in Fig. 1) is equipped with a 32-bit ARM Cortex-M3 MCU operating up to $24 \mathrm{MHz}$ and embedding 16-KB RAM and 256-KB eFlash ROM. It integrates also a $2.4 \mathrm{GHz}$ wireless transceiver compliant with the IEEE 802.15.4 standard. The featured MCU is optimized to provide high performance at very low power consumption. The WSN board is also equipped with an external antenna connector and 24 highly configurable GPIOs with Schmitt triggers inputs. Among the exposed GPIOs, the SDA and SCL lines, the ground pin (GND), and an output pin (DCI) have been used to implement the $\mathrm{I}^{2} \mathrm{C}$ communication with the RFID Section and provide power to the RFID chip.

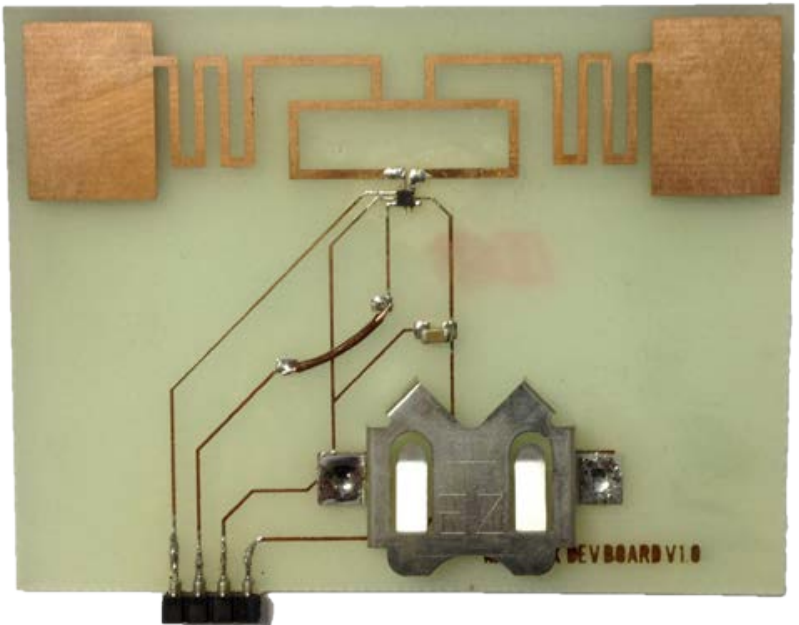

Fig. 2. Prototype of the developed RFID tag equipped with a small female header exposing the $\mathrm{I}^{2} \mathrm{C}$ interface (dimensions: $7.5 \times 5 \times 1.6 \mathrm{~cm}^{3}$ ).

\section{B. Software components}

Contiki OS [23] has been chosen to develop the firmware for the WSN node. It is a popular open-source operating system targeted to small microcontroller architectures and developed by the Swedish Institute of Computer Science. The Contiki communication stack is organized in several layers in which both protocol solutions and radio transceiver features are configured.

Contiki OS provides a full IP network stack, with standard IP protocols such as UDP, TCP, and HTTP, in addition to the new low-power standards. Specifically, Contiki supports also the recently standardized IETF protocols for low-power IPv6 networking, including the 6LowPAN [24] adaptation layer, the RPL IPv6 multi-hop routing protocol [25], and the CoAP RESTful application-layer protocol [26]. The IPv6 stack was contributed by Cisco and was, at the time of release, the smallest IPv6 stack to receive the IPv6 Ready certification. Furthermore, Contiki is highly memory efficient and provides a set of useful mechanisms for memory allocation. These features make Contiki the ideal choice for the development of new innovative smart applications, capable to exploit the new possibilities offered by the integration of RFID and WSN technologies.

In order to realize the proposed integration approach, the RFID based software components have been implemented in Contiki OS as system drivers. For this purpose, several functions to set hardware parameters, configure the communication interface and manage the memory of the Monza X-2K RFID chip have been implemented. Specifically, all functions required to read and write all the memory banks of the RFID chip have been designed by exploiting the $\mathrm{I}^{2} \mathrm{C}$ communication. As an example, Fig. 5(a) shows the $\mathrm{C}$ code used to read data from a specific memory location of the Monza X-2K chip while the related $\mathrm{I}^{2} \mathrm{C}$ signaling diagram is reported in Fig. 5(b). It takes as inputs the ID of the device from which to read data, the memory address from which to read data, the memory address from which to start reading, the address length in bytes, a pointer to the buffer used to store the data, and the number of bytes to read. 


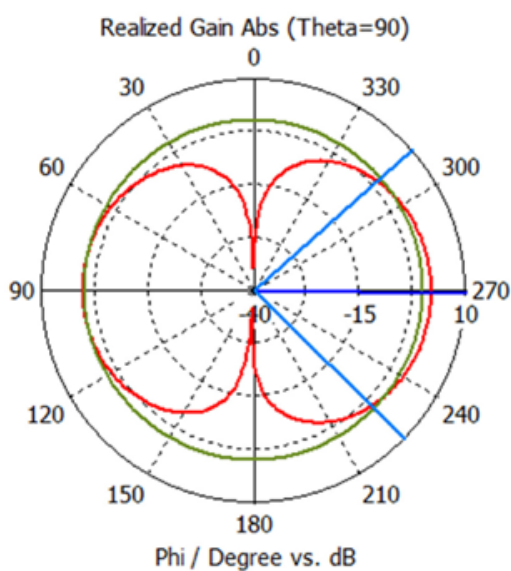

(a) E-plane polar radiation pattern

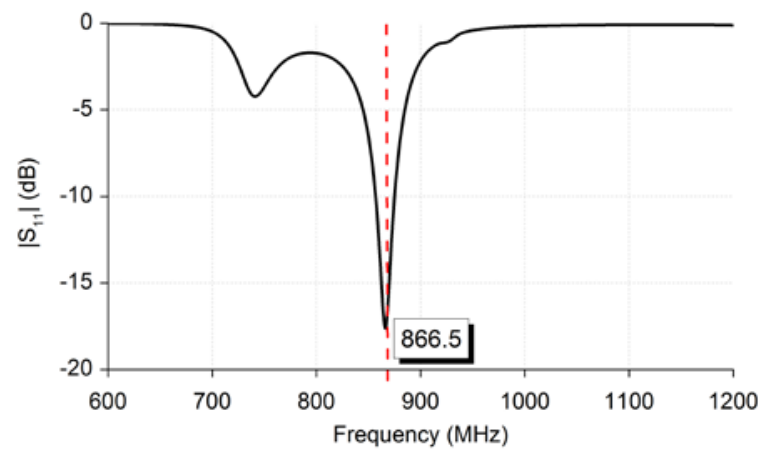

(b) Reflection coefficient

Fig. 3. Simulated radiation pattern and reflection coefficient for the designed dipole-like RFID antenna.

By implementing the $\mathrm{I}^{2} \mathrm{C}$ communication protocol, such a function saves the data in the specified buffer and returns the number of bytes read.

\section{EXPERIMENTATION}

In order to empirically analyze the proposed RFID-WSN integration approach and evaluate its performance in operating conditions, a series of experiments was carried out. The achieved results are presented and discussed below.

\section{A. Power consumption}

One of the fundamental aspects to investigate in the proposed RFID-WSN integration approach is the power consumption, being the limited on-board battery power a critical constraint when designing real-world WSN applications.

Therefore, in a first set of experiments, we aimed at measuring the impact of the $\mathrm{I}^{2} \mathrm{C}$-based communication (between the RFID tag and the WSN node) in terms of current consumption. To this end, we programmed the MCU of the WSN node to perform $\mathrm{I}^{2} \mathrm{C}$ read (write) transactions from (to) the user memory of the Monza X-2K RFID chip every 4 seconds. The DC voltage supplied to the RFID chip was fixed at $3 \mathrm{~V}$ by means of a power supply. In this way, the power consumption of the RFID chip depends solely on the DC current it absorbs. The measurement setup is sketched in Fig. 6: an Agilent DSO-X 2012A digital oscilloscope was used to

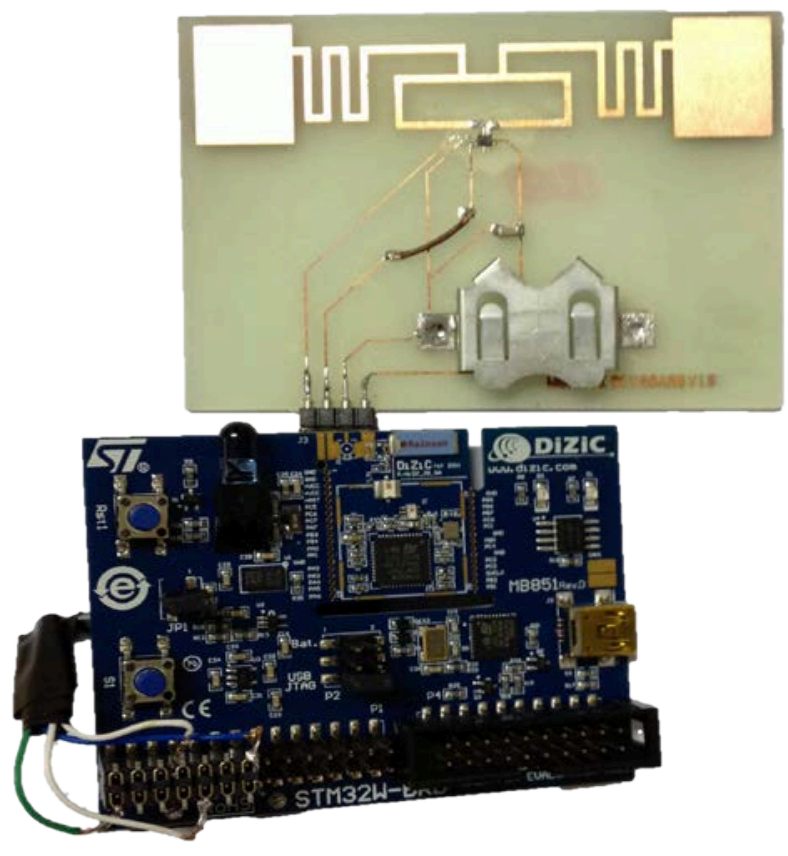

Fig. 4. Physical interconnection (via the I2C bus) between the developed RFID tag (RFID Section) and the ST Microelectronics MB954 (WSN Section).

track the voltage drop across a $1-\mathrm{k} \Omega$ precision shunt resistor specifically designed for current-sensing applications; then, the DC current flowing through the RFID chip was calculated by the Ohm's law.

int monzax_read(int i2c_device_id, int address, int address_len, uint8_t *data, int data_len)

\{

uint8_t i;

i2c_start();

/ Select monzax with write mode bit enabled */

i2c_write((i2c_device_id $<<1) \mid 0 \times 0)$;

/ Send address */

if (address_len $==1$ )

// 8-bit memory address

i2c_write(address \& 0xFF);

\} else \{

// 16-bit memory address

i2c write((address \& 0xFF00) >> 8);

\}

i2c_write(address \& 0xFF):

i2c_start();

/ Select monzax in read mode */

i2c_write((i2c_device_id $<<1) \mid 0 \times 1)$;

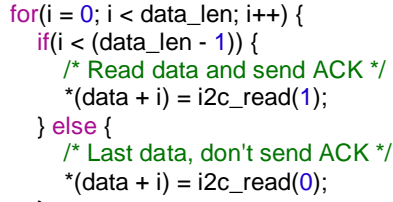

(a) Snippet $\mathrm{C}$ code for $\mathrm{I}^{2} \mathrm{C}$ read operations 


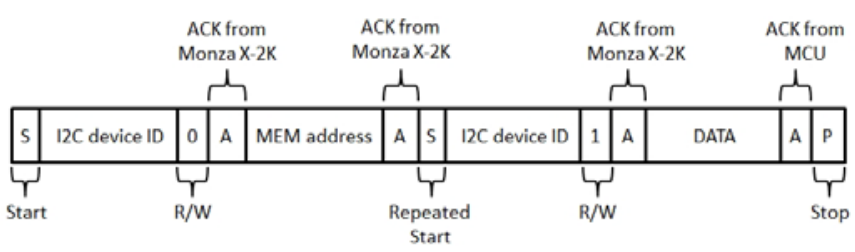

(b) $\mathrm{I}^{2} \mathrm{C}$ signaling diagram

Fig. 5. The snippet $\mathrm{C}$ code used to read data stored in a specific memory location of the Monza X-2K chip (a) and related signaling diagram of the $\mathrm{I}^{2} \mathrm{C}$ communication protocol (b).

The achieved results for the $\mathrm{I}^{2} \mathrm{C}$ write transactions are reported in Fig. 7. As shown, the maximum value recorded for the DC supply current is $140 \mu \mathrm{A}$, irrespective of the number of words transferred over the $\mathrm{I}^{2} \mathrm{C}$ bus - note that, according to the datasheet of the Monza X-2K chip, one word corresponds to 16 bits. The measured average times required to store data in the RFID chip user memory are approximately $50 \mathrm{~ms}, 190 \mathrm{~ms}$, and $400 \mathrm{~ms}$ respectively for 16-word, 50-word, and 136-word write operations - note that 136 words correspond to the entire user memory space of the Monza X-2K RFID chip.

As for the $\mathrm{I}^{2} \mathrm{C}$ read operations, a graphical representation similar to that in Fig. 7 is not required since constant values of both absorbed current (approximately $20 \mu \mathrm{A}$ ) and transaction duration (roughly $2 \mathrm{~ms}$ ) were recorded, regardless of the number of words transferred over the $\mathrm{I}^{2} \mathrm{C}$ bus. Such encouraging results in terms of current consumption spur the progress of this work towards the development of RF energy harvesting circuits [27], [28] in order to extract the required power directly from the RFID interrogation signal of the reader.

\section{B. RFID communication}

In a second set of experiments, we aimed at exploiting the RFID Gen2 air interface as a fully passive wake-up radio for the WSN node. In fact, when a WSN node is active and waiting to receive data, it wastes energy on idle listening and, in order to extend its lifetime, its radio could be turned off and its MCU set into a deep-sleep mode, ready to be woken up. Generally, there exist two approaches to wake up a WSN node: (1) duty cycling, where the node wakes up with a timer interrupt, and (2) wake-up radio, where the node wakes up with an external physical interrupt. In duty cycling, nodes are periodically set into the sleep mod e and therefore save a significant amount of energy at the expense of high latency for data delivery. On the contrary, the energy benefit of using radio wake-up is that nodes do not waste energy on idle listening, since they are only awakened when there is a request for transmission. This on-demand communication scheme also helps to decrease the packet latency, which is especially important for event-triggered network applications.

The possibility to selectively wake up WSN nodes using the Gen2 air interface is enabled by the Write Wake Up (WWU) mode provided by the Monza X-2K chip. This feature is tied to memory write operations performed over the RFID Gen2 interface. In order to enable this feature, we configured the interconnection between the RFID chip and the MCU of the WSN node as in Fig. 8. The MCU sets the Monza X-2K

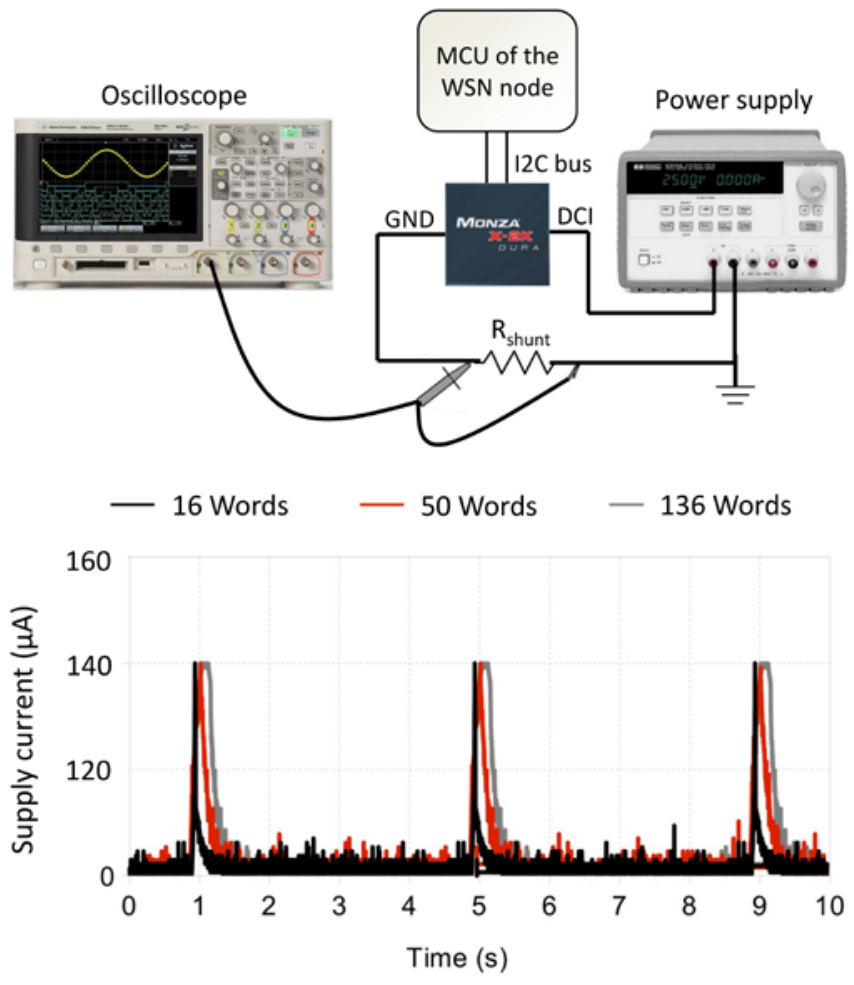

Fig. 7. Measured DC current absorbed by the Monza X-2K RFID chip during $\mathrm{I}^{2} \mathrm{C}$ write transactions (performed every 4 seconds) of 16,50 , and 136 usermemory words.

DCI pin to $0 \mathrm{~V}$ (sleep mode), forces the SCL and SDA lines of the $\mathrm{I}^{2} \mathrm{C}$ bus to remain high, and enters into a deep sleep mode. When the RFID reader performs a write operation, the Monza $\mathrm{X}-2 \mathrm{k}$ asserts the SCL IO by pulling the SCL line low for the duration of the write operation (approximately $4 \mathrm{~ms}$ ). This transition can then be detected by the sleeping MCU and used to wake up the system.

The maximum wake-up range was measured experimentally by using a commercial RFID Gen2 reader operating in the 865-868 MHz frequency band, set with 30-dBm transmit power, and configured to interrogate and write the user memory of the Monza X-2K chip at different distances. The experiments were conducted in a large lecture room with the reader antenna and the RFID-WSN node placed in the line of sight (LOS) $1.5 \mathrm{~m}$ above the floor, both oriented in the maximum-gain direction. The reader-node distance was increased in step of $0.5 \mathrm{~m}$ and, for each measurement point, the reader was instructed to perform 100 attempts to write the user memory of the RFID chip. The fraction of successful write operations, i.e. successful wake-ups of the WSN node, is plotted in Fig. 9 (black solid line). As shown, the success ratio remains above $80 \%$ up to $11 \mathrm{~m}$ before falling sharply to zero, thus demonstrating the effectiveness of leveraging the RFID Gen2 communications to passively wake up WSN nodes in real indoor scenarios.

In addition to the fully passive operation, the Monza X-2K RFID chip provides a battery-assisted passive (BAP) mode. In other words, both read sensitivity $(-17 \mathrm{dBm})$ and write sensitivity $(-12 \mathrm{dBm})$ of the chip are significantly boosted up to $-24 \mathrm{dBm}$ when a voltage is applied to the DCI pin. Using the same experimental setup of the previous experiment, we 


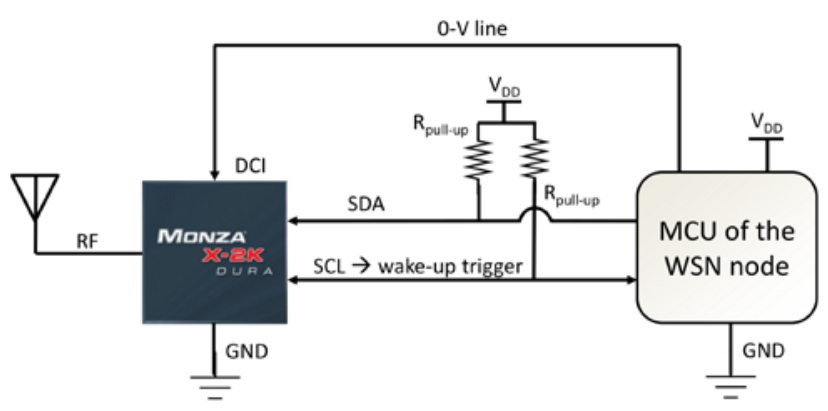

Fig. 8. Write Wake Up (WWU) mode: schematic of the interconnection between the Monza X-2K RFID chip and the MCU of the WSN node.

instructed the reader to perform, at each measurement point, 100 attempts to access the user memory of the RFID chip configured in BAP mode. The fraction of successful accesses is plotted in Fig. 9 (red dotted line). As shown, the success ratio remains above $80 \%$ up to $23 \mathrm{~m}$, which is a range quite comparable to that exhibited by the conventional IEEE 802.15.4 radio of WSN nodes. Moreover, the current absorbed by the Monza X-2K in BAP mode is just $25 \mu \mathrm{A}$ which is roughly three orders of magnitude less than WSN transceivers.

\section{Potential applications}

The extremely low power consumption and long operating range provided by the proposed integration approach make the hybrid RFID-WSN devices (referred to in the sequel as super motes) suitable for a wide range of applications. In the rest of this section, examples of potential applications, interesting mainly in the IoT context, enabled by the proposed RFIDWSN integration strategy are briefly summarized.

\section{A. Energy efficient protocols}

As previously described, the Write Wake Up (WWU) mode provided by the Monza X-2K chip provides super motes with an ultra-low-power RFI D-based wake-up radio. Such an operating mode allows to reduce substantially idle listening periods of WSN nodes, which represents the most prevalent source of energy waste. In fact, by using a secondary lowpower radio able to monitor the channel and detect possible communications, nodes can remain asleep for most of the time and activate their IEEE 802.15.4 radio transceiver only when a wake-up signal is triggered on the RFID Gen2 air interface. Despite the low cost of this solution, the high energy consumption of RFID readers makes the multi-hop wake-up mechanism unrealistic using this approach. However, these types of devices can be beneficial in scenarios where there is a mobile data sink or where the sensors are mobile (e. g., sensors on a person) and come in contact with a fixed sink, equipped with a reader, at some point [29]. For these scenarios, RFID readers could be used to send a specific command, which could either be multicast or targeted to a subset of nodes, to wake up only the super motes required to perform a given task. Since the RFID tag attached to a super mote does not consume energy when used in fully passive mode, such a mechanism has the potential to save a great amount of energy.

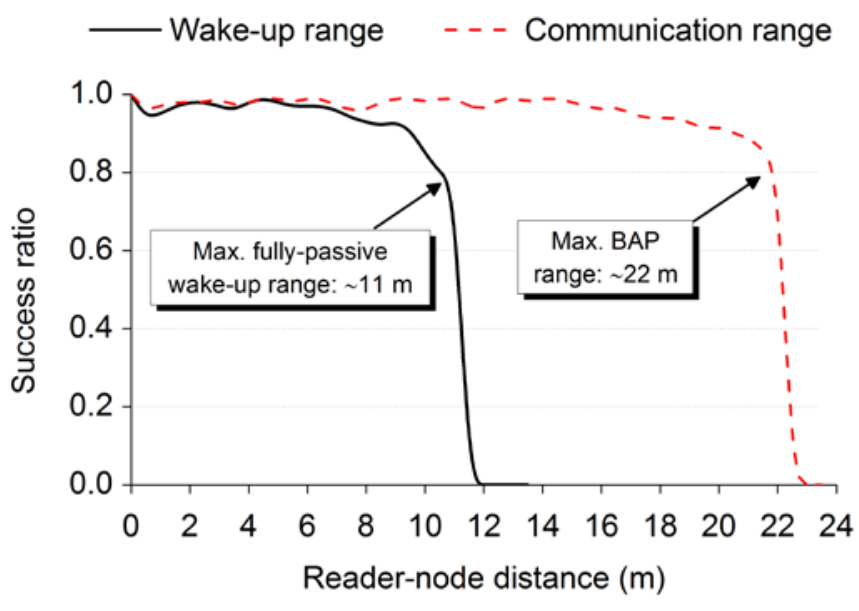

Fig. 9. Measured fraction of successful attempts to wake up the WSN node by exploiting the WWU mode (a) and to access to the Monza X-2K chip (configured in BAP mode) via the Gen2 air interface (b).

\section{B. Advanced supply chain management systems}

A simple yet interesting scenario enabled by the proposed RFID-WSN integration approach is shown in Fig. 10. It consists of a heterogeneous network made up of RFID readers and IEEE 802.15.4 gateways sharing the same backbone network. Such a network could be used to track and trace objects in an innovative supply chain management system, where RFID tags, WSN nodes, and super motes are distributed in the monitoring area to perform sensing, computation, and control tasks. In this scenario, super motes represent an added value because they are able to communicate with the overlay backbone network (last hop) via the zero-power RFID interface, and to interact among them in a multi hop fashion via the IEEE 802.15.4 protocol. Moreover, super motes still maintain the basic RFID functionality (i.e. the unique EPCglobal-standardized identification of the object the super mote is attached to).

\section{Smart parking systems}

Due to the rapid proliferation of vehicle availability, searching for a vacant parking space in a metropolitan area is becoming more and more difficult and it commonly results in traffic congestion and air pollution. To alleviate such a problem, smart parking systems, based on the use of novel technologies, have been proposed in the literature [30], [31]. However, most of them simply provide drivers with information (retrieved from deployed sensor networks) on the availability of parking spaces. The use of a heterogeneous RFID-WSN network could simplify the development of a reservation-based smart parking system, which could allow users to reserve a specific parking space and controllers to identify cars which improperly use reserved slots. Furthermore, super motes attached to each car could be selectively awakened by RFID readers located in the parking area, e.g. in order to gather specific environmental information, to check parking fee payments, etc.

\section{Other smart applications}

Note that, as discussed in [29], the proposed RFID-WSN integration approach enables many other interesting 


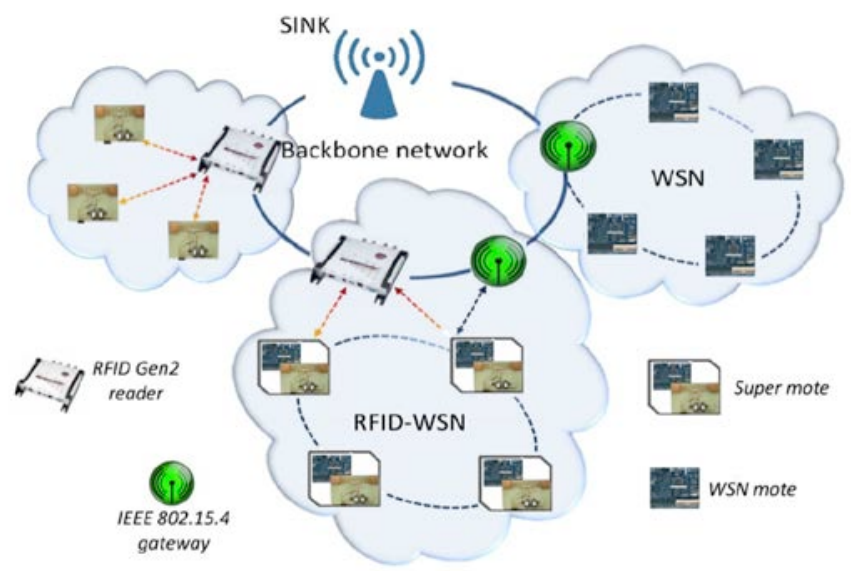

Fig. 10. Envisioned infrastructure of heterogeneous (RFID-WSN) wireless sensor networks.

applications, such as health monitoring systems, environmental sensing applications [32], and intelligent transportation systems. Finally, further improvements of our integration strategy could enable other and even smarter applications, which could benefit from the integration of RFID and WSN technologies.

\section{CONCLUSION}

A novel approach to integrate RFID and WSN technologies has been presented, discussed, and validated in this work. It relies on the physical interfacing of a new-generation, longrange, UHF RFID Gen2 tag with a conventional WSN node via the $\mathrm{I}^{2} \mathrm{C}$ communication bus. As a simple proof of concept, the capabilities provided by the proposed solution have been leveraged to implement a fully passive wake-up radio for WSN nodes with a maximum wake-up range of $11 \mathrm{~m}$ achieved in an indoor scenario. Furthermore, accurate measurements have demonstrated that our integration strategy makes the impact of the RFID section on the power consumption of the overall system negligible, thus preserving critical energyconstrained WSN applications.

As envisioned at the end of the paper, the extremely low power consumption and long operating range provided by the proposed RFID-WSN integration approach might find application in a wide array of IoT scenarios such as advanced supply chains, smart parking management services, health monitoring, environmental sensing, intelligent transportation.

\section{REFERENCES}

[1] L. Mainetti, L. Patrono, and A. Vilei: Evolution of wireless sensor networks towards the Internet of Things: a survey, 2011 International Conference on Software, Telecommunications and Computer Networks, SoftCOM 2011, pp. 16-21, 2011.

[2] L. Mainetti, L. Patrono, M.L. Stefanizzi, and R. Vergallo: An innovative and low-cost gapless traceability system of fresh vegetable products using RF technologies and EPCglobal standard, Computers and Electronics in Agriculture, Vol. 98, pp. 146-157, Oct. 2013.

[3] D. Alessandrelli, L. Mainetti, L. Patrono, G. Pellerano, M. Petracca, and M. L. Stefanizzi: Performance evaluation of an energy-efficient MAC scheduler by using a test bed approach, Journal of Communications Software and Systems, Vol. 9, No. 1, pp. 84-96, Mar. 2013.

[4] D. Alessandrelli, L. Mainetti, L. Patrono, G. Pellerano, M. Petracca, and M. L. Stefanizzi: Implementation and validation of an energy-efficient MAC scheduler for WSNs by a test bed approach, 2012 International
Conference on Software, Telecommunications and Computer Networks, SoftCOM 2012, Article number 6347615, 2012.

[5] L. Catarinucci, R. Colella, G. Del Fiore, L. Mainetti, V. Mighali, L. Patrono, and M.L. Stefanizzi: A cross-layer approach to minimize the energy consumption in wireless sensor networks, International Journal of Distributed Sensor Networks, Vol. 2014, Article number 268284, 2014.

[6] L. Catarinucci, S. Guglielmi, L. Mainetti, V. Mighali, L. Patrono, M.L. Stefanizzi, and L. Tarricone: An energy-efficient MAC scheduler based on a switched-beam antenna for wireless sensor networks, Journal of Communications Software and Systems, Vol. 9, No. 2, pp. 117-127, June 2013.

[7] L. Anchora, A. Capone, V. Mighali, L. Patrono, and F. Simone: A novel MAC scheduler to minimize the energy consumption in a Wireless Sensor Network, Ad Hoc Networks, Vol. 16, pp. 88-104, May 2014.

[8] M. Petracca, S. Bocchino, A. Azzarà, R. Pelliccia, M. Ghibaudi, and P. Pagano: WSN and RFID Integration in the IoT scenario: an Advanced safety System for Industrial Plants, Journal of Communications Software and Systems, Vol. 9, No. 1, pp. 104-113, Mar. 2013.

[9] C. Chen: Design of a Child Localization System on RFID and Wireless Sensor Networks, Journal of Sensors, Vol. 2010.

[10] Z. Xiong, F. Sottile, M. Spirito, and R. Garello: Hybrid Indoor Positioning Approaches Based on WSN and RFID, 4th IFIP International Conference on New Technologies, Mobility and Security, pp. 1-5, Feb. 2011.

[11] Z. Xiaoguang, and L. Wei: The research of network architecture in warehouse management system based on rfid and wsn integration, Proceedings of IEEE International Conference on Automation and Logistics, pp. 2556-2560, Sept. 2008

[12] R. Jurdak, A. Ruzzelli, and G. O'Hare: Multi-hop RFID wake-up radio: Design, evaluation and energy tradeoffs, 17th International Conference on Computer Communications and Networks (ICCCN), pp. 1 -8, 2008.

[13] H. Ba, I. Demirkol, and W. Heinzelman: Feasibility and benefits of passive RFID wake-up radios for wireless sensor networks, Proceedings of the IEEE Global Telecommunications Conference, pp. 1-5, 2010.

[14] A. P. Sample, D. J. Yeager, P. S. Powledge, and J. R. Smith: Design of an RFID-based battery-free programmable sensing platform, IEEE Transactions on Instrumentation and Measurement, Vol. 57, No. 11, pp. 2608-2615, Nov. 2008

[15] J. Polastre, R. Szewczyk, and D. Culler: Telos: Enabling Ultra-Low Power Wireless Research, International Conference on Information Sensor Networks (IPSN), pp. 364-369, 2005.

[16] D. De Donno, L. Catarinucci, and L. Tarricone: A Battery-Assisted Sensor-Enhanced RFID Tag Enabling Heterogeneous Wireless Sensor Networks, IEEE Sensors Journal, vol. 14, no. 4, pp. 1048-1055, Apr. 2014.

[17] Monza X-2K RFID IC [Online]. Available: http://www.impinj.com/

[18] Alien RFID [Online]. Available: http://www.alientechnology.com/tags/.

[19] D. De Donno, L. Catarinucci, A. Di Serio, and L. Tarricone: A longrange computational RFID tag for temperature and acceleration sensing applications, Progress in Electromagnetics Research C, vol. 45, pp. 223235,2013

[20] L. Catarinucci, S. Tedesco, D. De Donno, and L. Tarricone: Platformrobust passive UHF RFID tags: A case-study in robotics, Progress in Electromagnetics Research C, vol. 30, pp. 27-39, Aug. 2012.

[21] H. Lee, S. Kim, D. De Donno, and M. M. Tentzeris: A Novel Universal Inkjet-Printed EBG-backed Flexible RFID for Rugged On-Body and Metal Mounted Applications, 2012 IEEE MTT-S International Microwave Symposium Digest, pp. 1-4, 2012.

[22] STMicroelectronics, MB851 user manual [Online]. Available: http://www.st.com/.

[23] A. Dunkels, B. Gronvall, and T. Voigt: Contiki - a lightweight and flexible operating system for tiny networked sensors, IEEE Workshop on Embedded Networked Sensors, Nov. 2004.

[24] Y. Chen, H. Kun-mean, Z. Haiying, S. Hong-Ling, L. Xing, D. Xunxing, D. Hao, L. Jian-Jin, and C. de Vaulx: 6LoWPAN Stacks: A Survey, 7th International Conference on Wireless Communications, Networking and Mobile Computing (WiCOM), pp. 1-4, 2011.

[25] T. Clausen, U. Herberg, and M. Philipp: A critical evaluation of the IPv6 Routing Protocol for Low Power and Lossy Networks (RPL), IEEE 7th International Conference on Wireless and Mobile Computing, Networking and Communications (WiMob), pp. 365 - 372, Oct. 2011.

[26] C. Bormann, A.P. Castellani, and Z. Shelby: CoAP: An Application Protocol for Billions of Tiny Internet Nodes, IEEE Internet Computing, Vol. 16, No.2, pp. 62-67, 2012. 
[27] M. Russo, P. Solic, and M. Stella: Probabilistic modeling of harvested GSM energy and its application in extending UHF RFID tags reading range, Journal of Electromagnetic Waves and Applications, vol. 27, no. 4, 2013.

[28] D. De Donno, L. Catarinucci, and L. Tarricone: An UHF RFID energyharvesting system enhanced by a DC-DC charge pump in silicon-oninsulator technology, IEEE Microwave and Wireless Components Letters, vol. 23, no. 6, pp. 315-317, June 2013.

[29] H. Ba, I. Demirkol, and W. Heinzelman: Passive Wake-up Radios: From Devices To Applications, Ad Hoc Networks, Vol. 11, No. 8, pp. 26052621, Nov. 2013.

[30] S. V. Srikanth, P.J. Pramod, K.P. Dileep, S. Tapas, M.U. Patil, and C.B.N. Sarat: Design and Implementation of a Prototype Smart PARKing (SPARK) System Using Wireless Sensor Networks, International Conference on Advanced Information Networking and Applications Workshops, pp. 401 - 406, May 2009.

[31] Y. Jihoon, J. Portilla, and T. Riesgo: Smart parking service based on Wireless Sensor Networks, 38th Annual Conference on IEEE Industrial Electronics Society, pp. 6029-6034, 2012.

[32] D. De Donno, L. Catarinucci, and L. Tarricone: Enabling self-powered autonomous wireless sensors with new-generation $I^{2} C$-RFID chips, IEEE MTT-S International Microwave Symposium Digest, pp. 1-4, Jun. 2013.

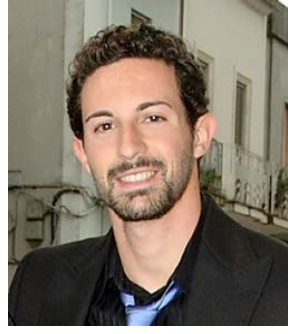

Danilo De Donno was born in Lecce, Italy, in 1983. He received the B.Sc. and M.Sc. degrees (cum laude) in telecommunication engineering from Politecnico di Milano, Italy, in 2005 and 2008, respectively, and the Ph.D. degree in information engineering from the University of Salento, Lecce, in 2012. In 2011, he was a Visiting Researcher with the School of Electrical and Computer Engineering, Georgia Institute of Technology, Atlanta, GA, USA. He is currently a Post-Doctoral Fellow with the Innovation Engineering Department, University of Salento. His research interests include the design of parallel algorithms on graphics processors, computational RFID systems, and software-defined radio experimentation.

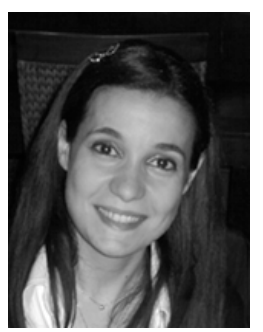

Maria Laura Stefanizzi graduated cum laude in Computer Engineering at University of Salento (Italy) in April 2012. Since January 2009 she collaborates with IDA Lab - IDentification Automation Laboratory at the Department of Innovation Engineering, University of Salento. Since July 2013 she is a $\mathrm{PhD}$ student at the University of Salento. Her activity is focused on the design and validation through test beds on real devices of innovative applications and protocols aimed to reduce power consumption in Wireless Sensor Networks. She is also involved in the study of new solutions for the integration of RFID and WSN technologies.

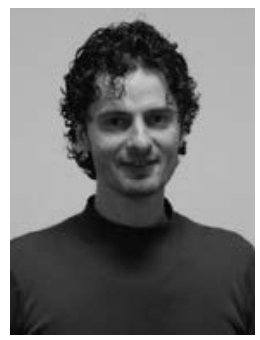

Luca Catarinucci is an Assistant Professor in Electromagnetic Fields at the Department of Innovation Engineering, University of Salento, Italy. His research activity is mostly focused on the implementation of electromagnetic simulation tools, in the FDTD analysis of human-antenna interaction and in the electromagnetic characterisation of materials. Further contributions deal with the time domain reflectometry (TDR) for the characterisation of fluids and in the radiofrequency identification (RFID) antenna and system design. He authored more than 50 papers on international and national journals and in international and national conferences and a chapter of a book with international diffusion.

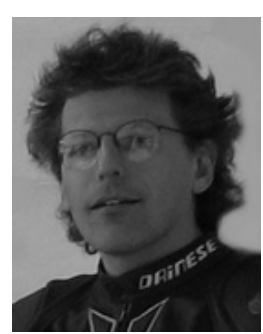

Luca Mainetti is an associate professor of software engineering and computer graphics at the University of Salento. His research interests include web design methodologies, notations and tools, services oriented architectures and IoT applications, and collaborative computer graphics. He is a scientific coordinator of the GSA Lab - Graphics and Software Architectures Lab and IDA Lab - IDentification Automation Lab at the Department of Innovation Engineering, University of Salento. He is the Rector's delegate at the ICT.

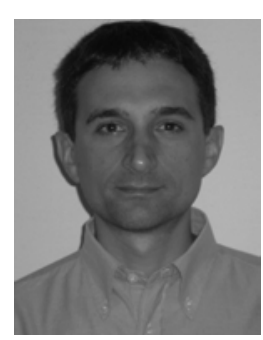

Luigi Patrono received his MS in Computer Engineering from University of Lecce, Lecce, Italy, in 1999 and $\mathrm{PhD}$ in Innovative Materials and Technologies for Satellite Networks from ISUFI-University of Lecce, Lecce, Italy, in 2003. He is an Assistant Professor of Network Design at the University of Salento, Lecce, Italy. His research interests include RFID, EPCglobal, Internet of Things, Wireless Sensor Networks, and design and performance evaluation of protocols. He is Organizer Chair of the international Symposium on RFID Technologies and Internet of Things within the IEEE SoftCOM conference. He is author of about 70 scientific papers published on international journals and conferences and four chapters of books with international diffusion.

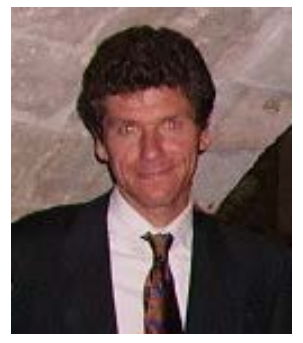

Luciano Tarricone received the Laurea degree (cum laude) in electronic engineering and the Ph.D. degree from Rome University La Sapienza, Rome, Italy, in 1989 and 1994, respectively. Since 1994, he has been a Researcher with the University of Perugia, Italy, and since 1998, he has been a Professore Incaricato of EM fields and EM compatibility. Since 2001, he has been a Faculty Member with the Department of Innovation Engineering, University of Salento, Lecce, Italy, where he is a Full Professor of electromagnetic fields. He has authored over 300 scientific papers. His main contributions are in the modeling of microscopic interactions of EM fields and biosystems, and in numerical methods for efficient computer-aided design (CAD) of microwave circuits and antennas. He is currently involved in bioelectromagnetics, EM energy harvesting and wireless power transmission, novel CAD tools and procedures for MW circuits, RFID, and EM high-performance computing. 